\title{
Neoplasm of mixed mesenchymal and neuroepithelial origin : liposarcomatous meningioma combined with gliomas ${ }^{1}$
}

\author{
SAMRUAY SHUANGSHOTI \\ From the Department of Pathology, Faculty of Medicine, Chulalongkorn University, \\ Bangkok, Thailand
}

SUMMARY A case is reported of an intracranial neoplasm of mixed mesenchymal and neuroepithelial (glial) origin occurring in the parieto-parasagittal region of the brain of a 19 year old man. A meningioma with liposarcomatous and psammomatous components comprised the mesenchymal part of the combined tumour. The neuroepithelial portion was composed of mixed ependymoma and astrocytoma. The combined neoplasm had the gross appearances and several microscopic features of giant-celled glioblastoma, giant cell sarcoma, and monstrocellular sarcoma. The findings suggest that these tumours have a mixed mesenchymal and neuroepithelial origin.

Some primary tumours of the neuraxis may contain adipose tissue in whole or in part, as in lipoma (Zettner and Netsky, 1960) or in meningioma with a lipomatous component (Bailey and Bucy, 1931). Dublin (1967) briefly mentioned that liposarcoma is an exceedingly rare type of meningeal sarcoma but he did not illustrate an example. Caldwell and Zinninger (1925) described a man who had an extradural pure liposarcoma of the lumbosacral spinal canal. This tumour arose from the fifth lumbar and the first sacral vertebrae, and extended into the dural sac covering the cauda equina. A man reported by Berger (1928) had a liposarcoma of the right cerebellopontine angle. The neoplasm extended through the ipsilateral jugular foramen to form both intracranial and extracranial masses. A liposarcoma of a girl recorded by Kothandaram (1970) was adherent to the under surface of the dura mater over the cerebral convexity. The tumour covered and depressed the cerebral hemispheres, eroded the skull at both parietal regions, and extended through the craniotomy defect into the deep aspects of the scalp. To the best of the author's knowledge, the case to be presented below is the only example of

1 Supported by a research grant of Chulalongkorn University on the Royal Silver Jubilee Anniversary, 1971. intracranial meningioma containing a liposarcomatous component combined with a mixed glioma.

\section{CASE REPORT}

One year before hospitalization, this 19 year old Thai male labourer experienced occasional diffuse headaches but he could work regularly. One month before admission, he first suffered from twitching of the left upper and lower extremities lasting about five minutes but recovered spontaneously. This symptom reappeared two weeks later. Two days before admission, the same episode recurred and lasted longer but it again disappeared spontaneously.

At the time of hospitalization, there were no physical abnormalities. Radiographs of the skull and thorax, and routine studies of the blood and urine revealed normal findings. Brain scan and carotid angiogram, however, gave evidence of a mass lesion in the right parieto-parasagittal region which was confirmed at craniotomy. A demarcated tumour resembling a meningioma was not adherent to the dura mater; it was completely removed. Postoperatively, the patient suffered left hemiparesis which gradually improved during the ensuing three months. One year later there was only slight weakness on the left side of the body and the patient could do daily work as usual. At the time of this report, three years after the craniotomy, he was without neurological deficits. 


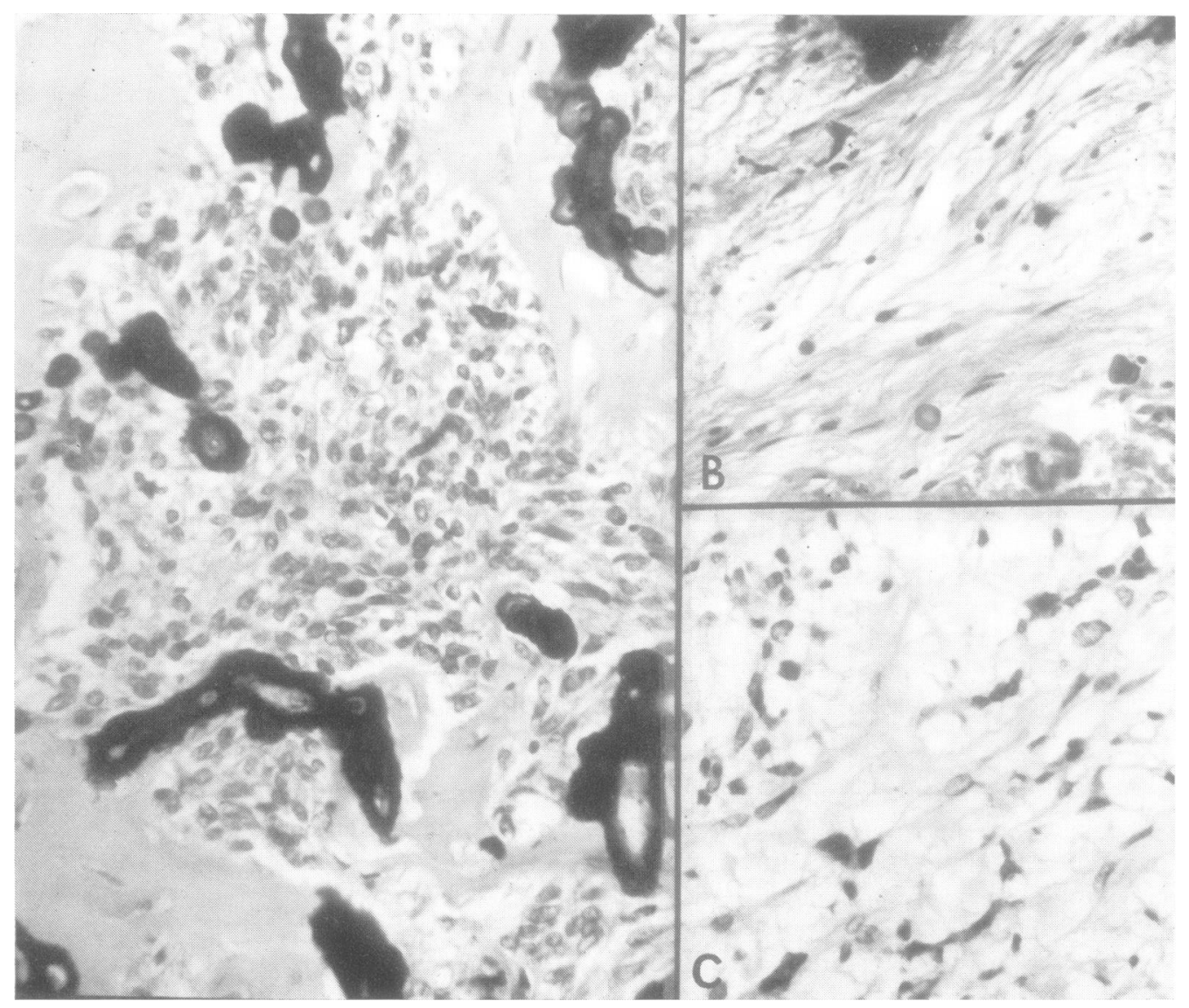

FIG. 1A (above). A cluster of meningocytes, many calcific plaques, psammoma bodies, and severely hyalinized bands of the connective tissue are present in the tumour. $H$ and $E, \times 300 . \mathrm{B}$. Fibroblasts and a few calcific areas are shown. $H$ and $E, \times 225$. C. Stellate-shaped mesenchymal cells are arranged in loose mesh. $H$ and $E, \times 350$.

The specimen of a circumscribed grey and nodular tumour weighing $65 \mathrm{~g}$ was partly soft and partly calcified extensively (Chulalongkorn Hosp. Surg. Path. No. 12-3859). It was fixed in $10 \%$ formalin and then embedded in paraffin. The following stains were used: haematoxylin and eosin (H and E), Mallory's phosphotungstic acid haematoxylin, Wilder's method for reticulin fibres, and toluidine blue (Nissl). Frozen sections were done on some fragments fixed in formalin, and stained with Sudan IV.

Microscopically, the tumour consisted of multiple types of cell. In one place, a desmoplastic and extensively calcified zone containing a few psammoma bodies was surrounded by sheets of numerous neoplastic cells of variable size; one type (Fig. 1A) was ovoid and syncytial and considered to be meningocytes. The other type was spindle-shaped and interpreted as fibroblasts (Fig. 1B). Occasional stellate-shaped cells considered to be mesenchymal cells (Fig. 1C) were also mingled. There were, furthermore, many large and bizarre cells having weakly acidophilic and foamy cytoplasm as seen in $\mathrm{H}$ and $\mathrm{E}$ preparations (Fig. 2). However, cytoplasmic droplets of sudanophilic material, presumably neutral fat, were noted in the sections stained with Sudan IV. Their large irregular and hyperchromatic nuclei contained occasional inclusion bodies. These bizarre $N$ giant cells were interpreted as lipoblasts. Many $N$ reticulin fibres were scattered in this portion of the tumour. 


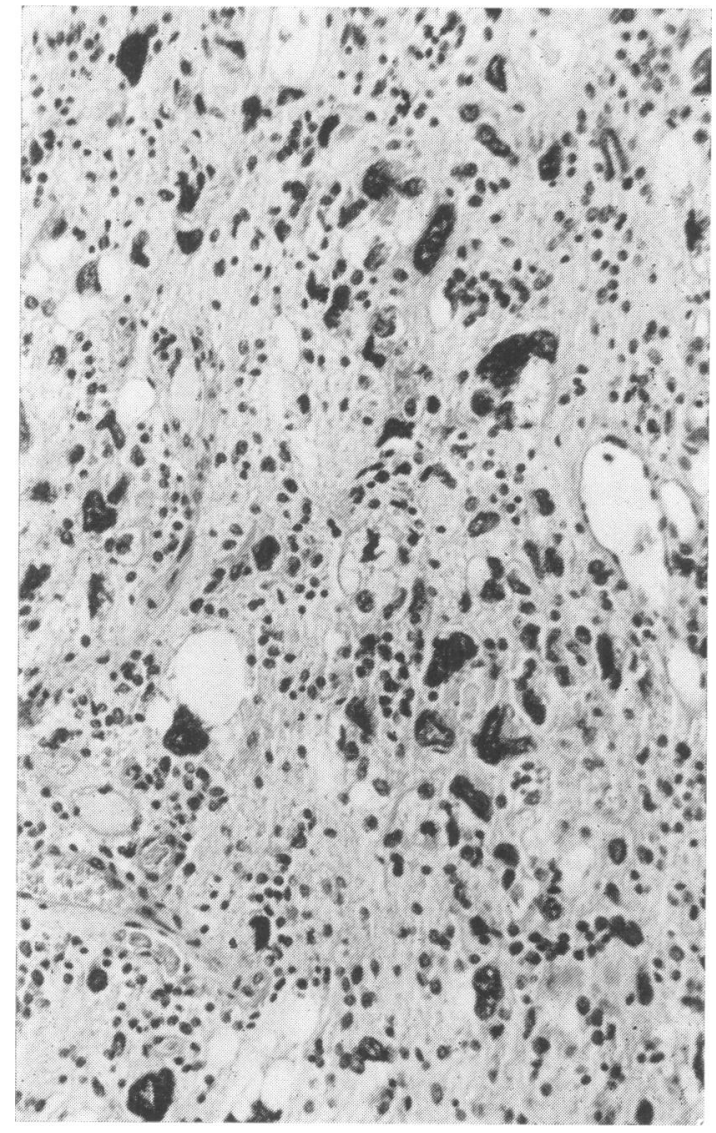

There were, in addition, many carrot-shaped tumour cells having ovoid and vesicular nuclei. They were often arranged radially around small blood vessels. Their fine polar processes frequently were attached to the adventitia forming pseudorosettes. A few true rosettes were also present. These cells were considered to be neoplastic ependymal cells (Fig. 3A). The lumena of several blood vessels in this part of the tumour were obliterated by proliferated endothelial cells (Fig. 3B) and by recently-formed thrombi. Reticulin fibres were few in this zone of the neoplasm (Fig. 3C).

Another type of less numerous cells had a polygonal shape, glassy cytoplasm, and small vesicular eccentric nuclei; some cells had a few short coarse processes extending from their angular borders. They were interpreted as plump astrocytes (Fig. 4). Tigroid substances were not seen in any tumour cells.

The diagnosis was mixed mesenchymal and neuroepithelial tumour (combined mesenchymal,
FIG. 2. Many bizarre lipoblasts with vacuolated cytoplasm and irregular hyperchromatic nuclei are disseminated at random. $H$ and $E, \times 160$.

meningocytic, and fibroblastic meningioma with liposarcomatous and psammomatous components, and with ependymoma and astrocytoma) of the right parieto-parasagittal region.

\section{DISCUSSION}

The diverse morphological features of this tumour raise the question as to whether it is a purely mesenchymal or neuroepithelial (glial) neoplasm. The parasagittal location, the circumscribed outline and nodular surface, the presence of meningocytes, fibroblasts, psammoma bodies, and reticulin fibres in the fibroblastic areas are all features diagnostic of meningioma. However, the presence of ependymal cells with true rosettes and pseudorosettes, astrocytes, vascular endothelial hyperplasia, and the lack of reticulin fibres among these neoplastic cells are indicative 


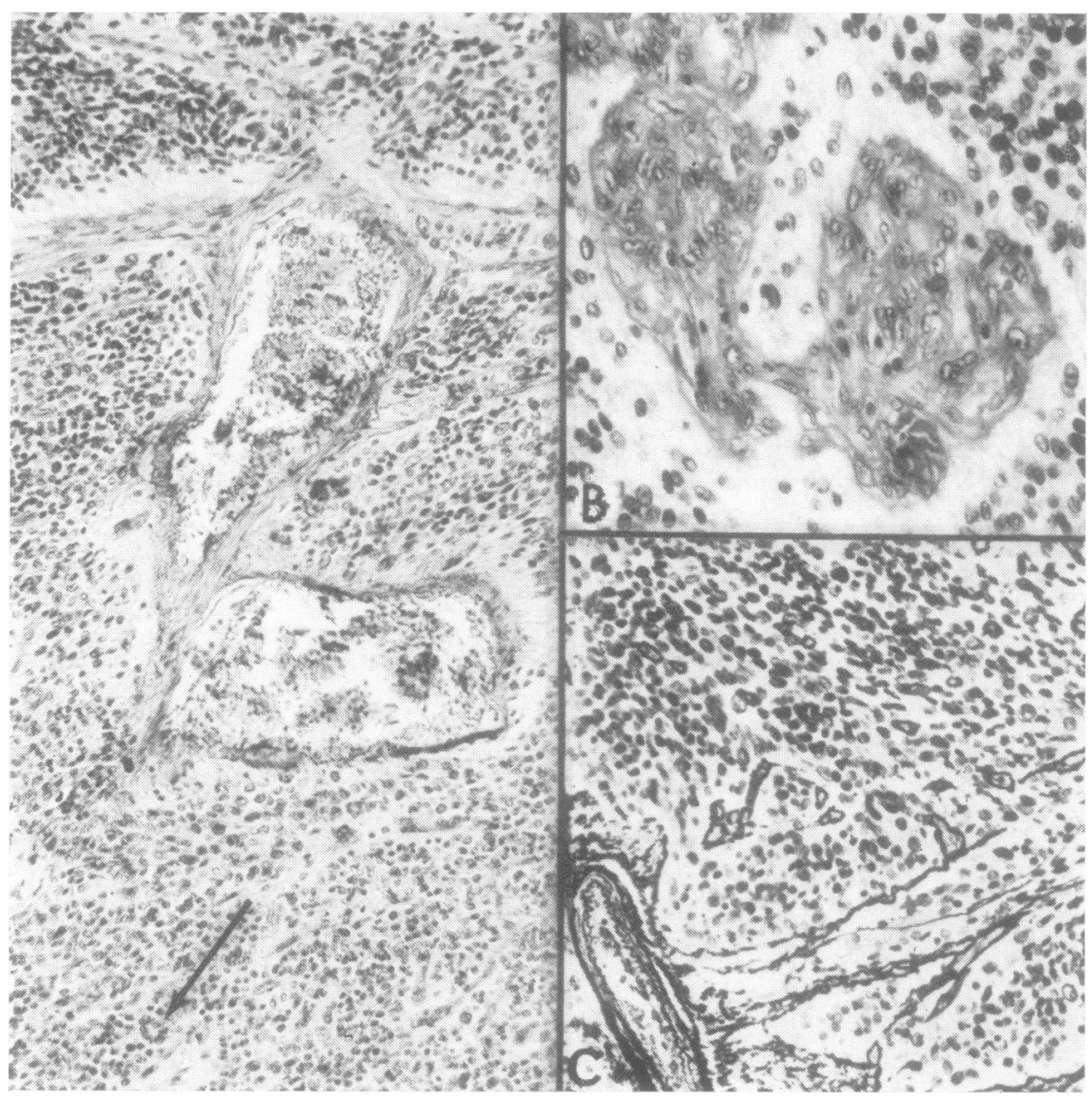

FIG. 3A (above). Area of ependymoma showing numerous tumour cells having fine polar processes attaching to the vascular adventitia forming pseudorosettes. The arrow points to a distinctive true rosette. $H$ and $E, \times 140 . \mathrm{B}$. Blood vessels are occluded by proliferated endothelial cells, and are surrounded by ependymal neoplastic cells. $H$ and $E, \times 250$. C. Sheet of ependymal tumour cells traversed by a few reticulin fibres, some of which are adherent to the vascular adventitia. Wilder's reticulin stain, $\times 160$.

of glioma. A unitary diagnosis does not describe all cellular constituents in this tumour. The author, therefore, considers the diagnosis of mixed meningioma, ependymoma, and astrocytoma to be most appropriate.

Components of meningioma are diverse because of multipotential behaviour of this mesenchymal type of neoplasm (Bailey and Bucy, 1931; Willis, 1948). It is, therefore, not surprising to encounter a liposarcomatous component in the mixed mesenchymal and neuroepithelial tumour when meningioma is part of this combined neoplasm.
The present tumour, however, must be distinguished from other neoplasms of multiple cellular populations. Teratoma, the growth:usually located in the vicinity of the midline of 3 . the body as is the present tumour, may contain 8 many cellular types. A diencephalic teratoma reported by Levin (1942) had islands of bone, 음 cartilage, squamous epithelium, ependymal cells, $>$ and glioblastoma multiforme. Only adipose tissue? in the present tumour, however, is foreign to the N brain; hence this mass is not a teratoma (Willis, $N$ 1948).

Extracranial liposarcoma tends to metastasize ${ }_{0}^{\text {W }}$ 


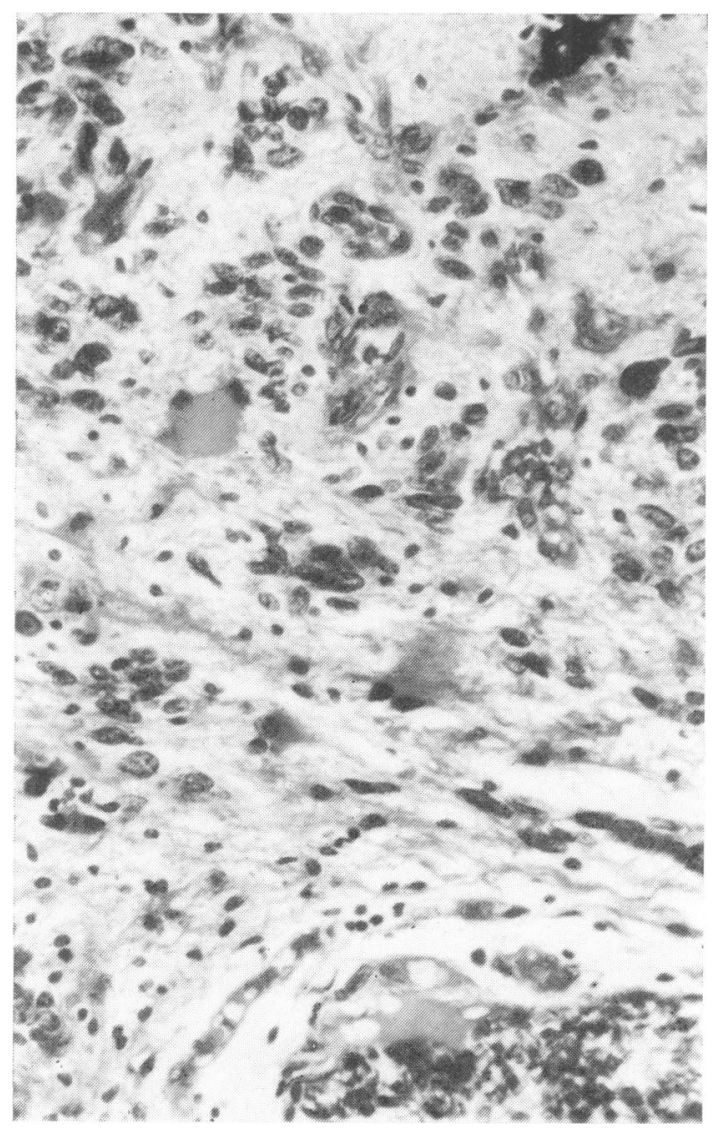

to the brain (Fender, 1933). Metastatic extracranial liposarcoma to an intracranial glioma, therefore, must be excluded. However, there was no evidence of any malignant neoplasm outside the neuraxis in a complete physical examination as well as in follow-up study of the patient for three years. The symptoms and signs of the disease in this patient were entirely cerebral.

The circumscribed outline of this tumour, and the presence of spindle-shaped cells, bizarre giant cells with intranuclear inclusion bodies and cytoplasmic vacuoles, many reticulin fibres in some parts of the mass, and numerous blood vessels are also findings diagnostic of the 'giantcelled or magnocellular glioblastoma' (Alcott and McCort, 1962; Russell and Rubinstein, 1963), or 'giant cell sarcoma' (Kernohan and Uihlein, 1962), or 'monstrocellular sarcoma' (Zülch, 1965). Although the presence of vascular
FIG. 4. A few plump neoplastic astrocytes with homogeneous cytoplasm and eccentric vesicular nuclei are present among many other tumour cells. $H$ and $E, \times 280$. endothelial proliferation in the 'giant-celled glioblastoma' was denied by Russell and Rubinstein (1963), it was observed by Becker, Benyo, and Roessmann (1967). The present tumour, therefore, might be diagnosed as 'giantcelled glioblastoma'. The author, however, has considered the latter, the 'giant cell sarcoma', and the 'monstrocellular sarcoma' as neoplasms of mixed mesenchymal and neuroepithelial type (Shuangshoti and Netsky, 1971a, b). The present tumour, therefore, is not distinguished from these 'giant cell tumours', and further illustrates the combined mesenchymal and neuroepithelial nature of these neoplasms. The tissue of origin and the mode of occurrence of these mixed tumours have been discussed (Shuangshoti and Netsky, 1971a).

Our patient had a favourable postoperative survival despite the neoplasm containing a lipo- 
sarcomatous component. Characteristically, the tumours of mixed mesenchymal and neuroepithelial variety have sharply circumscribed outlines (Shuangshoti and Netsky, 1971a, b). This macroscopic feature may allow, in some patients, total extirpation with satisfactory result. Cases have been recorded of 'monstrocellular sarcoma' with postoperative survival of seven years without radiation or chemotherapy (Garcia-Bengochea and Collins, 1969). Another patient has been asymptomatic for 27 years after removal of a mixed meningioma, neurilemmoma, astrocytoma, and oligodendroglioma from the frontal lobe (Shuangshoti, Netsky, and Jane, 1971).

I am grateful to Dr. Somsak Dhechakaisaya, Professor and Head of the Department of Pathology; and Dr. Chaturaporn Hongsaprabhas, Assistant Professor of Surgery, the Division of Neurosurgery, Chulalongkorn University, who gave permission to report this case.

\section{REFERENCES}

Alcott, D. L., and McCort, J. J. (1962). Magnocellular glioblastoma. Cancer Seminar, 3, 52-55.

Bailey, P., and Bucy, P. C. (1931). The origin and nature of meningeal tumours. American Journal of Cancer, 15, 15-54.

Becker, D. P., Benyo, R., and Roessmann, U. (1967). Glial origin of monstrocellular tumour. Case report of prolonged survival. Journal of Neurosurgery, 26, 72-77.

Berger, W. (1929). Über einen eigenartigen Tumor (lipoplastisches Sarkom) der Schädelbasis mit Einbruch in das Gehörorgan. Beiträge zur Aratomie, Physiologie, Patholo- gie und Therapie des Ohres, der Nase und des Halses, 27, 301-324.

Caldwell, J. A., and Zinninger, M. M. (1925). Extradural liposarcoma of the spinal canal. A clinical and pathological report. Surgery, Gynecology and Obstetrics, 40, 476480.

Dublin, W. B. (1967). Fundamentals of Neuropathology. 2nd edn, p. 324. Thomas: Springfield, Ill.

Fender, F. A. (1933). Liposarcoma. Report of a case with intracranial metastases. American Journal of Pathology, 9, 909-914.

Garcia-Bengochea, F., and Collins, G. H. (1969). Monstrocellular sarcoma of the brain: 6-year postoperative survival. Case report. Journal of Neurosurgery, 31, 686-689.

Kernohan, J. W., and Uihlein, A. (1962). Sarcomas of the Brain, pp. 70-89. Thomas: Springfield, Ill.

Kothandaram, P. (1970). Dural liposarcoma associated with subdural hematoma. Case report. Journal of Neurosurgery, 33, 85-87.

Levin, P. M. (1942). Glioblastoma arising in a hypothalamic teratoid and invading the neurohypophysis. Journal of Neuropathology and Experimental Neurology, 1, 146-157.

Russell, D. S., and Rubinstein, L. J. (1963). Pathology of Tumours of the Nervous System, 2nd edn., pp. 154-155. Arnold: London.

Shuangshoti, S., and Netsky, M. G. (1971a). Neoplasms of mixed mesenchymal and neuroepithelial origin. Relation to 'monstrocellular sarcoma' or 'giant-celled glioblastoma'. Journal of Neuropathology and Experimental o Neurology, 30, 290-309.

Shuangshoti, S., and Netsky, M. G. (1971b). Brain tumor of $w$ mixed mesenchymal and neuroepithelial origin. Cas $\mathrm{V}$ report. Journal of Neurosurgery, 34, 808-813.

Shuangshoti, S., Netsky, M. G., and Jane, J. A. (1971). Neo̊ 을 plasms of mixed mesenchymal and neuroepithelial type $\overrightarrow{-}$ With consideration to the relationship between menir gioma and neurilemmoma. Journal of the Neurologic\& Sciences, 14, 277-291.

Willis, R. A. (1948). Pathology of Tumours, pp. 718-728 an 940-984. Butterworth: London.

Zettner, A., and Netsky, M. G. (1960). Lipoma of the corpus callosum. Journal of Neuropathology and Experimental Neurology, 19, 305-319.

Zülch, K. J. (1965). Brain Tumors. Their Biology and Pathology, 2nd edn., pp. 219-225. Springer: New York. 